\title{
ON THE VOLUME SET OF POINT SETS IN VECTOR SPACES OVER FINITE FIELDS
}

\author{
LE ANH VINH \\ (Communicated by Jim Haglund)
}

\begin{abstract}
We show that if $\mathcal{E}$ is a subset of the $d$-dimensional vector space over a finite field $\mathbb{F}_{q}(d \geq 3)$ of cardinality $|\mathcal{E}| \geq(d-1) q^{d-1}$, then the set of volumes of $d$-dimensional parallelepipeds determined by $\mathcal{E}$ covers $\mathbb{F}_{q}$. This bound is sharp up to a factor of $(d-1)$, as taking $\mathcal{E}$ to be a $(d-1)$-hyperplane through the origin shows.
\end{abstract}

\section{INTRODUCTION}

Let $q$ be an odd prime power, and let $\mathbb{F}_{q}$ be a finite field of $q$ elements. The distribution of the determinant of matrices with entries in a restricted subset of $\mathbb{F}_{q}$ has been studied recently by various researchers (see, for example, [1, 2, 5, 6] and the references therein). In particular, Covert et al. 2 studied this problem in a more general setting. They examined the distribution of volumes of $d$ dimensional parallelepipeds determined by a large subset of $\mathbb{F}_{q}^{d}$. More precisely, for any $\boldsymbol{x}^{1}, \ldots, \boldsymbol{x}^{d} \in \mathbb{F}_{q}^{d}$, define $\operatorname{vol}\left(\boldsymbol{x}^{1}, \ldots, \boldsymbol{x}^{d}\right)$ as the determinant of the matrix whose rows are $\boldsymbol{x}^{j}$ s. The focus of $[2$ is to study the cardinality of the volume set

$$
\operatorname{vol}(\mathcal{E})=\left\{\operatorname{vol}\left(\boldsymbol{x}^{1}, \ldots, \boldsymbol{x}^{d}\right): \boldsymbol{x}^{j} \in \mathcal{E}\right\} .
$$

A subset $\mathcal{E} \subseteq \mathbb{F}_{q}^{d}$ is called a product-like set if $\left|\mathcal{E} \cap \mathcal{H}_{n}\right| \ll|\mathcal{E}|^{n / d}$ for any $n$ dimensional subspace $\mathcal{H}_{n} \subset \mathbb{F}_{q}^{d}$. Covert et al. [2] showed that if $\mathcal{E} \subseteq \mathbb{F}_{q}^{d}$ is a product-like set of cardinality $|\mathcal{E}| \gg q^{15 / 8}$, then $\mathbb{F}_{q}^{*} \subseteq \operatorname{vol}(\mathcal{E})$. When $\mathcal{E} \subseteq \mathbb{F}_{q}^{3}$ is an arbitrary set, they obtained the following result.

Theorem 1.1 ([2, Theorem 2.10]). Suppose that $\mathcal{E} \subseteq \mathbb{F}_{q}^{3}$ of cardinality $|\mathcal{E}| \geq C q^{2}$ for a sufficiently large constant $C>0$. There exists $c>0$ such that

$$
|\operatorname{vol}(\mathcal{E})| \geq c q \text {. }
$$

In this short note, we show that under the same condition, $\mathcal{E}$ determines all possible volumes. More precisely, we will prove the following general result.

Theorem 1.2. When $\mathcal{E} \subseteq \mathbb{F}_{q}^{d}$ and $|\mathcal{E}| \geq(d-1) q^{d-1}, \operatorname{vol}(\mathcal{E})=\mathbb{F}_{q}$.

Remark 1.3. The assumption $|\mathcal{E}| \geq(d-1) q^{d-1}$ is sharp up to a factor of $(d-1)$, as taking $\mathcal{E}$ to be a $(d-1)$-hyperplane through the origin shows.

Received by the editors October 1, 2011 and, in revised form, December 5, 2011.

2010 Mathematics Subject Classification. Primary 11 T99.

This research is supported by the Vietnam National Foundation for Science and Technology Development, grant No. 101.01-2011.28. 
Note that the implied constant in the symbol ' $\gg$ ' may depend on the integer parameter $d$. We recall that the notation $U \gg V$ is equivalent to the assertion that $|U| \geq c|V|$ holds for some constant $c>0$.

\section{Preparations}

Recall that

$$
\operatorname{vol}\left(\boldsymbol{x}^{1}, \ldots, \boldsymbol{x}^{d}\right)=\boldsymbol{x}^{1} \cdot\left(\boldsymbol{x}^{2} \wedge \ldots \wedge \boldsymbol{x}^{d}\right),
$$

where the dot product is defined by the usual formula

$$
\boldsymbol{u} \cdot \boldsymbol{v}=u_{1} v_{1}+\ldots+u_{d} v_{d} .
$$

The generalized cross product, also called the wedge product, is given by the identity

$$
\boldsymbol{u}^{2} \wedge \ldots \wedge \boldsymbol{u}^{d}=\operatorname{det}\left(\begin{array}{c}
\boldsymbol{i} \\
\boldsymbol{u}^{2} \\
\ldots \\
\boldsymbol{u}^{d}
\end{array}\right)
$$

where $\boldsymbol{i}=\left(\boldsymbol{i}^{1}, \ldots, \boldsymbol{i}^{d}\right)$ indicates the coordinate directions in $\mathbb{F}_{q}^{d}$.

2.1. Geometric incidence estimates. One of our main tools is a two-set version of the geometric incidence estimate developed by D. Hart, A. Iosevich, D. Koh, and M. Rudnev in [4] (see also 3] for an earlier version and 2] for a function version of this estimate). Note that going from one-set formulation in the proof of [4, Theorem 2.1] to a two-set formulation is just a matter of inserting a different letter into a couple of places.

Lemma 2.1 ([4, Theorem 2.1]). Let $B(\cdot, \cdot)$ be a nondegenerate bilinear form in $\mathbb{F}_{q}^{d}$. For any $\mathcal{E}, \mathcal{F} \subseteq \mathbb{F}_{q}^{d}$, let

$$
\nu_{t, B}(\mathcal{E}, \mathcal{F})=\sum_{B(\boldsymbol{x}, \boldsymbol{y})=t} \mathcal{E}(\boldsymbol{x}) \mathcal{F}(\boldsymbol{y}),
$$

where here and throughout the paper $\mathcal{E}(\boldsymbol{x})$ denotes the characteristic function of $\mathcal{E}$. We have

$$
\nu_{t, B}(\mathcal{E}, \mathcal{F})=|\mathcal{E}||\mathcal{F}| q^{-1}+R_{t, B}(\mathcal{E}, \mathcal{F})
$$

where

$$
\left|R_{t, B}(\mathcal{E}, \mathcal{F})\right|^{2} \leq|\mathcal{E}||\mathcal{F}| q^{d-1}, \text { if } t \neq 0 .
$$

As a corollary of Lemma 2.1, D. Hart and A. Iosevich [3] derived the following result.

Corollary $2.2([\underline{3}, 4])$. For any $\mathcal{E}, \mathcal{F} \subseteq \mathbb{F}_{q}^{d}$, let

$$
\mathcal{E} \cdot \mathcal{F}=\{\boldsymbol{u} \cdot \boldsymbol{v}: \boldsymbol{u} \in \mathcal{E}, \boldsymbol{v} \in \mathcal{F}\} .
$$

We have $\mathbb{F}_{q}^{*} \subseteq \mathcal{E} \cdot \mathcal{F}$ when $|\mathcal{E}||\mathcal{F}| \gg q^{d+1}$.

We also need the following corollary.

Corollary 2.3. Let $B(\cdot, \cdot)$ be a nondegenerate bilinear form in $\mathbb{F}_{q}^{d} \times \mathbb{F}_{q}^{d}$. For any $\mathcal{E} \subset \mathbb{F}_{q}^{d} \backslash(0, \ldots, 0)$, let

$$
B^{*}(\mathcal{E})=\{B(\boldsymbol{x}, \boldsymbol{y}): \boldsymbol{x}, \boldsymbol{y} \in \mathcal{E}\} \backslash\{0\}
$$


We have

$$
\left|B^{*}(\mathcal{E})\right| \geq q\left(1-\frac{q+q^{3 / 2}}{|\mathcal{E}|+q^{3 / 2}}\right) .
$$

Proof. For any $\boldsymbol{x} \in \mathcal{E}$, there exist at most $q$ vectors $\boldsymbol{y}$ such that $\boldsymbol{x} \cdot \boldsymbol{y}=0$. Hence,

$$
\sum_{t \in \mathbb{F}_{q}^{*}} \nu_{t, B}(\mathcal{E}, \mathcal{E}) \geq|\mathcal{E}|^{2}-q|\mathcal{E}|
$$

Lemma 2.1 implies that

$$
\nu_{t, B}(\mathcal{E}, \mathcal{E}) \leq \frac{|\mathcal{E}|^{2}}{q}+q^{1 / 2}|\mathcal{E}|,
$$

for any $t \in \mathbb{F}_{q}^{*}$. The corollary follows immediately from (2.1) and (2.2).

2.2. Cross-product set. Let $\mathcal{H}$ be the $d$-dimensional vector space over a finite field $\mathbb{F}_{q}$. Let $\left\{\boldsymbol{v}^{1}, \ldots, \boldsymbol{v}^{d}\right\}$ be an orthogonal basis of $\mathcal{H}$. For any $d$ vectors $\boldsymbol{u}^{1}, \ldots, \boldsymbol{u}^{d}$ $\in \mathcal{H}$, each vector $\boldsymbol{u}^{i}$ can be written uniquely as a linear combination of $\left\{\boldsymbol{v}^{1}, \ldots, \boldsymbol{v}^{d}\right\}$, i.e.

$$
\boldsymbol{u}^{i}=\sum_{j=1}^{d} u_{j}^{i} \boldsymbol{v}^{j}, \quad u_{j}^{i} \in \mathbb{F}_{q}, 1 \leq j \leq d
$$

We have

$$
\boldsymbol{u}^{1} \wedge \boldsymbol{u}^{2} \wedge \ldots \wedge \boldsymbol{u}^{d}=\operatorname{det}\left(\left(u_{j}^{i}\right)_{i, j=1}^{d}\right) \boldsymbol{v}^{1} \wedge \ldots \wedge \boldsymbol{v}^{d}
$$

For any $\mathcal{E} \subseteq \mathcal{H}$, define

$$
\mathcal{D}_{\mathcal{E}, d}^{*}:=\left\{\operatorname{det}\left(\left(u_{j}^{i}\right)_{i, j=1}^{d}\right): \boldsymbol{u}^{i}=\sum_{j=1}^{d} u_{j}^{i} \boldsymbol{v}^{j} \in \mathcal{E}, 1 \leq i \leq d\right\} \backslash\{0\} .
$$

For any $\boldsymbol{x} \in \mathbb{F}_{q}^{d}$ and $\mathcal{E} \subseteq \mathbb{F}_{q}^{d}$, let

$$
g_{\mathcal{E}}(\boldsymbol{x})=\#\left\{\left(\boldsymbol{u}^{1}, \ldots, \boldsymbol{u}^{d-1}\right) \in \mathcal{E}^{d-1}: \boldsymbol{u}^{1} \wedge \ldots \wedge \boldsymbol{u}^{d-1}=\boldsymbol{x}\right\} .
$$

Define the cross-product set of $\mathcal{E}$ :

$$
\mathcal{F}_{\mathcal{E}}^{*}=\left\{\boldsymbol{x} \in \mathbb{F}_{q}^{d}: g_{\mathcal{E}}(\boldsymbol{x}) \neq 0\right\} \backslash\{(0, \ldots, 0)\} .
$$

For any $\boldsymbol{x} \in \mathbb{F}_{q}^{d} \backslash\{(0, \ldots, 0)\}$, let $\mathcal{H}^{\boldsymbol{x}}:=\boldsymbol{x}^{\perp}=\left\{\boldsymbol{y} \in \mathbb{F}_{q}^{d}: \boldsymbol{x} \cdot \boldsymbol{y}=0\right\}$. It is clear that $\boldsymbol{x} \in \mathcal{F}_{\mathcal{E}} \backslash\{(0, \ldots, 0)\}$ if and only if there exist $\boldsymbol{u}^{1}, \ldots, \boldsymbol{u}^{d-1} \in \mathcal{H}^{\boldsymbol{x}} \cap \mathcal{E}$ such that

$$
\boldsymbol{u}^{1} \wedge \ldots \wedge \boldsymbol{u}^{d-1}=\boldsymbol{x} .
$$

It follows from (2.3), (2.4), and (2.5) that

$$
\mathcal{F}_{\mathcal{E}}^{*} \cap\left\{l \boldsymbol{x}: l \in \mathbb{F}_{q}^{*}\right\}=\mathcal{D}_{\mathcal{E} \cap \mathcal{H}^{x}, d-1}^{*} .
$$

Hence, we have proved the following lemma.

Lemma 2.4. For any $\mathcal{E} \subseteq \mathbb{F}_{q}^{d}$, we have

$$
\left|\mathcal{F}_{\mathcal{E}}^{*}\right|=\sum_{H \in G(d-1, d)}\left|\mathcal{D}_{\mathcal{E} \cap H, d-1}^{*}\right|
$$

where $G(d-1, d)$ is the set of all $(d-1)$-dimensional subspaces of $\mathbb{F}_{q}^{d}$. 


\section{Proof of Theorem 1.2}

The proof proceeds by induction. We first consider the base case, $d=3$. We show that if $|\mathcal{E}|>2 q^{2}$, then the cross-product set $\mathcal{F}_{\mathcal{E}}^{*}$ is large. From Lemma 2.4. we have

$$
\left|\mathcal{F}_{\mathcal{E}}^{*}\right|=\sum_{\mathcal{H} \in G(2,3)}\left|\mathcal{D}_{\mathcal{E} \cap \mathcal{H}, 2}^{*}\right|
$$

Since each non-zero vector lies in $(q+1)$ two-dimensional subspaces of $\mathbb{F}_{q}^{2}$,

$$
\sum_{\mathcal{H} \in G(2,3)}|\mathcal{E} \cap \mathcal{H}|=(q+1)|\mathcal{E}| .
$$

Let

$$
G_{(2,3)}^{\mathcal{E}}=\{\mathcal{H} \in G(2,3):|\mathcal{E} \cap \mathcal{H}|>q\}
$$

we have

$$
\sum_{\mathcal{H} \in G_{(2,3)}^{\mathcal{E}}}|\mathcal{E} \cap \mathcal{H}|>(q+1)|\mathcal{E}|-q|G(2,3)|=(q+1)|\mathcal{E}|-q\left(q^{2}+q+1\right)>q^{3} .
$$

Corollary 2.3 implies that

$$
\left|\mathcal{D}_{\mathcal{E} \cap \mathcal{H}, 2}^{*}\right| \geq q\left(1-\frac{q+q^{3 / 2}}{|\mathcal{E} \cap \mathcal{H}|+q^{3 / 2}}\right),
$$

for any $\mathcal{H} \in G(2,3)$. Since

$$
f(x)=1-\frac{q+q^{3 / 2}}{x+q^{3 / 2}}
$$

is a concave function on $\left[q, q^{2}\right]$,

$$
\begin{aligned}
\sum_{\mathcal{H} \in G_{(2,3)}^{\mathcal{E}}}\left|\mathcal{D}_{\mathcal{E} \cap \mathcal{H}, 2}^{*}\right| & \geq q \sum_{\mathcal{H} \in G_{(2,3)}^{\mathcal{E}}}\left(1-\frac{q+q^{3 / 2}}{|\mathcal{E} \cap \mathcal{H}|+q^{3 / 2}}\right) \\
& \geq q \frac{\sum_{\mathcal{H} \in G_{(2,3)}^{\mathcal{E}}}|\mathcal{E} \cap \mathcal{H}|}{q^{2}}\left(1-\frac{q+q^{3 / 2}}{q^{2}+q^{3 / 2}}\right) \\
& >q^{2}\left(1-q^{-1 / 2}\right)>q^{2} / 2 .
\end{aligned}
$$

It follows from (3.1) and (3.2) that $\left|\mathcal{F}_{\mathcal{E}}^{*}\right|>q^{2} / 2$. Hence, $|\mathcal{E}|\left|\mathcal{F}_{\mathcal{E}}^{*}\right|>q^{4}$. Corollary 2.2 implies that $\mathbb{F}_{q}^{*} \subseteq \mathcal{E} \cdot \mathcal{F}_{\mathcal{E}}^{*} \subseteq \operatorname{vol}(\mathcal{E})$. By choosing a matrix of identical rows, we have $0 \in \operatorname{vol}(\mathcal{E})$. The base case $d=3$ follows.

Suppose that the theorem holds for $d-1 \geq 3$; we show that it also holds for $d$. Similarly, we show that if $|\mathcal{E}|>(d-1) q^{d-1}$, then the cross-product set $\mathcal{F}_{\mathcal{E}}^{*}$ is large. Since each non-zero vector lies in $\left(q^{d-1}-1\right) /(q-1)(d-1)$-dimensional subspaces of $\mathbb{F}_{q}^{d}$,

$$
\sum_{\mathcal{H} \in G(d-1, d)}|\mathcal{E} \cap \mathcal{H}|=\frac{q^{d-1}-1}{q-1}|\mathcal{E}| .
$$

Let

$$
G_{(d-1, d)}^{\mathcal{E}}=\left\{\mathcal{H} \in G(d-1, d):|\mathcal{E} \cap \mathcal{H}|>(d-2) q^{d-2}\right\}
$$


We have

$$
\begin{aligned}
\sum_{\mathcal{H} \in G_{(d-1, d)}^{\mathcal{E}}}|\mathcal{E} \cap \mathcal{H}| & >\frac{q^{d-1}-1}{q-1}|\mathcal{E}|-(d-2) q^{d-2}|G(d-1, d)| \\
& =\frac{\left(q^{d-1}-1\right)|\mathcal{E}|-(d-2) q^{d-2}\left(q^{d}-1\right)}{q-1} \\
& >q^{d},
\end{aligned}
$$

when $q$ is sufficiently large (in fact, $q>d$ is enough). Since $|\mathcal{E} \cap \mathcal{H}| \leq q^{d-1}$ for each $\mathcal{H} \in G_{(d-1, d)}^{\mathcal{E}}$,

$$
\left|G_{(d-1, d)}^{\mathcal{E}}\right|>q^{d} / q^{d-1}=q .
$$

By induction hypothesis, for any $\mathcal{H} \in G_{(d-1, d)}^{\mathcal{E}}$,

$$
\left|\mathcal{D}_{\mathcal{E} \cap \mathcal{H}, d-1}^{*}\right|=q-1 .
$$

Putting (3.3), (3.4) and Lemma 2.4 together, we have

$$
\left|\mathcal{F}_{\mathcal{E}}^{*}\right|=\sum_{\mathcal{H} \in G(d-1, d)}\left|\mathcal{D}_{\mathcal{E} \cap \mathcal{H}, d-1}^{*}\right|>\sum_{\mathcal{H} \in G_{(d-1, d)}^{\mathcal{E}}}\left|\mathcal{D}_{\mathcal{E} \cap \mathcal{H}, d-1}^{*}\right|>q(q-1)>q^{2} / 2 .
$$

Hence, $|\mathcal{E}|\left|\mathcal{F}_{\mathcal{E}}^{*}\right|>q^{d+1}$. Corollary 2.2 implies that $\mathbb{F}_{q}^{*} \subseteq \mathcal{E} \cdot \mathcal{F}_{\mathcal{E}}^{*} \subseteq \operatorname{vol}(\mathcal{E})$. By choosing a matrix of identical rows, we have $0 \in \operatorname{vol}(\mathcal{E})$. This completes the proof of the theorem.

\section{REFERENCES}

1. O. Ahmadi and I. E. Shparlinski, Distribution of matrices with restricted entries over finite fields, Inda. Mathem. 18(3) (2007), 327-337. MR.2373685(2008k:11127)

2. D. Covert, D. Hart, A. Iosevich, D. Koh, and M. Rudnev, Generalized incidence theorems, homogeneous forms and sum-product estimates in finite fields, European Journal of Combinatorics, 31 (2010), 306-319. MR2552610 (2010m:11014)

3. D. Hart and A. Iosevich, Sums and products in finite fields: an integral geometric viewpoint, Radon transforms, geometry, and wavelets, Contemporary Mathematics, 464, Amer. Math. Soc. (2008). MR2440133 (2009m:11032)

4. D. Hart, A. Iosevich, D. Koh, and M. Rudnev, Averages over hyperplanes, sum-product theory in vector spaces over finite fields and the Erdös-Falconer distance conjecture, Trans. Amer. Math. Soc., 363 (2011), 3255-3275. MR2775806(2012e:42008)

5. L. A. Vinh, Distribution of determinant of matrices with restricted entries over finite fields, Journal of Combinatorics and Number Theory, 1(3) (2009), 203-212. MR2681305 (2011g:11056)

6. L. A. Vinh, Singular matrices with restricted rows in vector spaces over finite fields, Discrete Mathematics, 312(2) (2012), 413-418. MR2852600

7. L. A. Vinh, On the permanents of matrices with restricted entries over finite fields, SIAM J. Discrete Mathematics, 26(3) (2012), 997-1007. MR.3022119

University of Education, Vietnam National University, Hanoi, Vietnam

E-mail address: vinhla@vnu.edu.vn 